\title{
ISBN Group Numbers
}

978-0

Namibia ......................................... NA

978-0 + 978-1

Australia ..........................................

Bermuda ..................................... BM

Canada - English Language .............. CA

Gibraltar .......................................... G

Ireland .............................................. IE

New Zealand .................................. NZ

South Africa .................................. ZA

Swaziland ...................................... SZ

United Kingdom..............................GB

United States ..................................... US

Zimbabwe .................................... ZW

978-2

Belgium - French speaking .............. BE

Canada - French Language................ CA

France............................................FR

Luxembourg .................................... LU

Switzerland.......... CH

978-3

Austria

Germany

Luxembourg

Switzerland

978-4

Japan

978-5

Armenia

Azerbaijan

Belarus

Kazakhstan ................................... KZ

Latvia .............................................. LV

Lithuania .......................................... LT

Russian Federation ......................... RU

Ukraine ...........................................

978-7

China ................................................

978-80

Czech Republic ................................ CZ

Slovakia......................................... SK

978-81

India............................................... IN

978-82

Norway .............................................

978-83

Poland .............................................PL

978-84

Spain ............................................. ES

978-85

Brazil............................................... BR

978-86

Bosnia and Herzegovina.................. BA

Croatia...........................................HR

Kosova.......................................... KV

Montenegro .......................................ME
Serbia

Slovenia.....

Srpska, Republic of

978-87

Denmark

978-88

Italy....

978-89

Korea, Republic of

978-90

Netherlands

978-91

Sweden

978-92

European Union Organizations ........ LU

International NGO Publishers ...........GB

978-93

India

978-94

Netherlands .. IN

978-600

Iran, Islamic Republic of

978-601

Kazakhstan

978-602

Indonesia........................................ ID

978-603

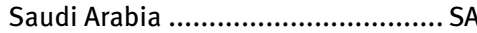

978-604

Viet Nam ..................................... VN

978-605

Turkey ........................................... TR

978-606

Romania .......................................RO

978-607

Mexico ........................................... MX

978-608

Macedonia, The Former Yugoslav Republic of

978-609

Lithuania

978-611

Thailand.

978-612

Peru

978-613

Mauritius ......................................... MU

978-614

Lebanon......................................... LB

978-615

Hungary ...........................................

978-616

Thailand........................................ $\mathrm{TH}$

978-617

Ukraine .........................................

978-618

Greece
978-619

Bulgaria .........................................

978-620

Mauritius ......................................... MU

978-621

Philippines........................................

978-950

Argentina ..................................... AR

978-951 + 978-952

Finland ............................................

978-953

Croatia.............................................HR

978-954

Bulgaria ..........................................

978-955

Sri Lanka............................................K

978-956

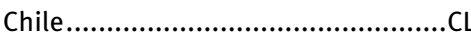

978-957

Taiwan ............................................TW

978-958

Colombia ............................................

978-959

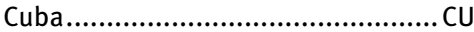

978-960

Greece ......................................... GR

978-961

Slovenia........................................... SI

978-962

Hong Kong ..................................... HK

978-963

Hungary........................................... HU

978-964

Iran, Islamic Republic of................... IR

978-965

Israel ...................................................IL

978-966

Ukraine ............................................ UA

978-967

Malaysia ........................................MY

978-968

Mexico ............................................. MX

978-969

Pakistan.......................................... PK

978-970

Mexico ............................................ MX

978-971

Philippines.......................................PH

978-972

Portugal............................................PT

978-973

Romania .......................................RO

978-974

Thailand........................................ TH

978-975

Turkey........................................ TR 
978-976

Caribbean Community

978-977

Egypt ............................................ EG

978-978

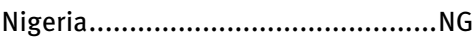

978-979

Indonesia........................................ ID

978-980

Venezuela, Bolivarian Republic of .... VE

978-981

Singapore …...................................

978-982

South Pacific ........................................F

978-983

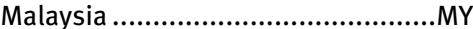

978-984

Bangladesh...................................... BD

978-985

Belarus ......................................... BY

978-986

Taiwan .............................................TW

978-987

Argentina .................................... AR

978-988

Hong Kong .................................... HK

978-989

Portugal.............................................PT

978-9926

Bosnia and Herzegovina.................. BA

978-9927

Qatar ...............................................QA

978-9928

Albania ..............................................

978-9929

Guatemala ...................................... GT

978-9930

Costa Rica ........ CR

978-9931

Algeria ........................................... DZ

978-9932

Lao People‘s Democratic Republic.... LA

978-9933

Syrian Arab Republic...................... SY

978-9934

Latvia .............................................. LV

978-9935

Iceland …...................................... IS

978-9936

Afghanistan ....................................AF

978-9937

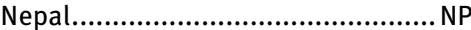

978-9938

Tunisia ............................................ TN

978-9939

Armenia .....AM

978-9940

Montenegro .......................................

978-9941

Georgia
978-9942

Ecuador

EC

978-9943

Uzbekistan........................................ UZ

978-9944

Turkey.

TR

978-9945

Dominican Republic

..DO

978-9946

Korea, Democratic People‘s

Republic of

KP

978-9947

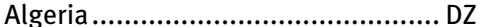

978-9948

United Arab Emirates

... AE

978-9949

Estonia

978-9950

Palestine, State of

..EE

978-9951

Kosova.

978-9952

Azerbaijan

978-9953

Lebanon.

978-9954

Morocco

978-9955

Lithuania

978-9956

Cameroon

978-9957

Jordan

978-9958

Bosnia and Herzegovina.................. BA

978-9959

Libya

978-9960

Saudi Arabia

978-9961

Algeria

978-9962

Panama

978-9963

Cyprus

978-9964

Ghana.

978-9965

Kazakhstan .................................... KZ

978-9966

Kenya

978-9967

Kyrgyzstan ...................................... KG

978-9968

Costa Rica ....................................... CR

978-9970

Uganda

978-9971

Singapore

SG
978-9972

Peru

PE

978-9973

Tunisia .......................................... TN

978-9974

Uruguay ............................................ UY

978-9975

Moldova, Republic of ...................... MD

978-9976

Tanzania, United Republic of .............TZ

978-9977

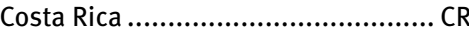

978-9978

Ecuador ........................................... EC

978-9979

Iceland ........................................... IS

978-9980

Papua New Guinea...........................PG

978-9981

Morocco .......................................... MA

978-9982

Zambia .......................................... ZM

978-9983

Gambia

GM

978-9984

Latvia

LV

978-9985

Estonia ..........................................EE

978-9986

Lithuania .......................................... LT

978-9987

Tanzania, United Republic of .............TZ

978-9988

Ghana.

978-9989

Macedonia, The Former Yugoslav

Republic of

MK

978-99901

Bahrain

978-99902

Gabon (reserved)

978-99903

Mauritius

978-99904

Curaçao

CW

978-99905

Bolivia, Plurinational State of ..........BO

978-99906

Kuwait ............................................ KW

978-99908

Malawi.............................................WW

978-99909

Malta ....

978-99910

Sierra Leone.....................................SL

978-99911

Lesotho

978-99912

Botswana

BW 


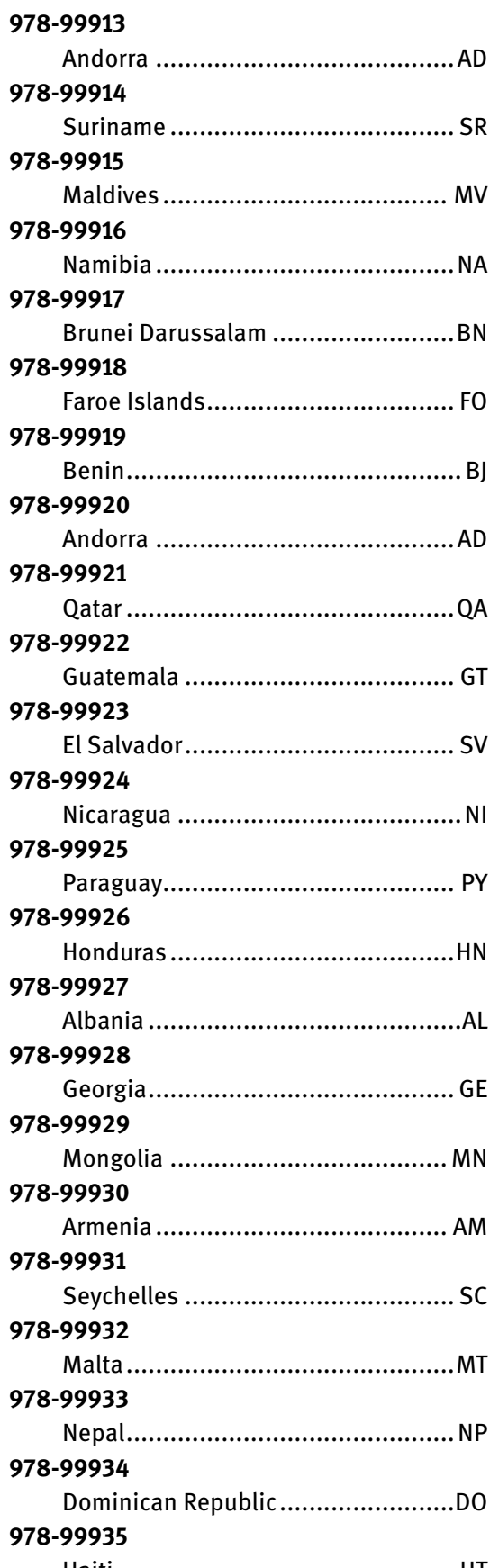

Haiti .

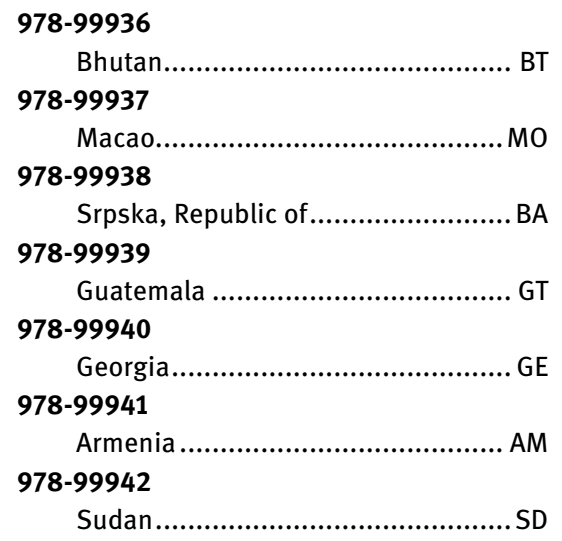

978-99943

978-99944

Ethiopia ............................................ET

978-99945

Namibia ........................................ NA

978-99946

Nepal.........................................NP

978-99947

Tajikistan ...................................... TJ

978-99948

Eritrea........................................... ER

978-99949

Mauritius ..........................................MU

978-99950

Cambodia ......................................... KH

978-99951

Congo, The Democratic Republic

of the.....

978-99952

Mali.

978-99953

Paraguay.

978-99954

Bolivia, Plurinational State of ...........BO

978-99955

Srpska, Republic of

. BA

978-99956

Albania

...AL

978-99957

Malta

MT

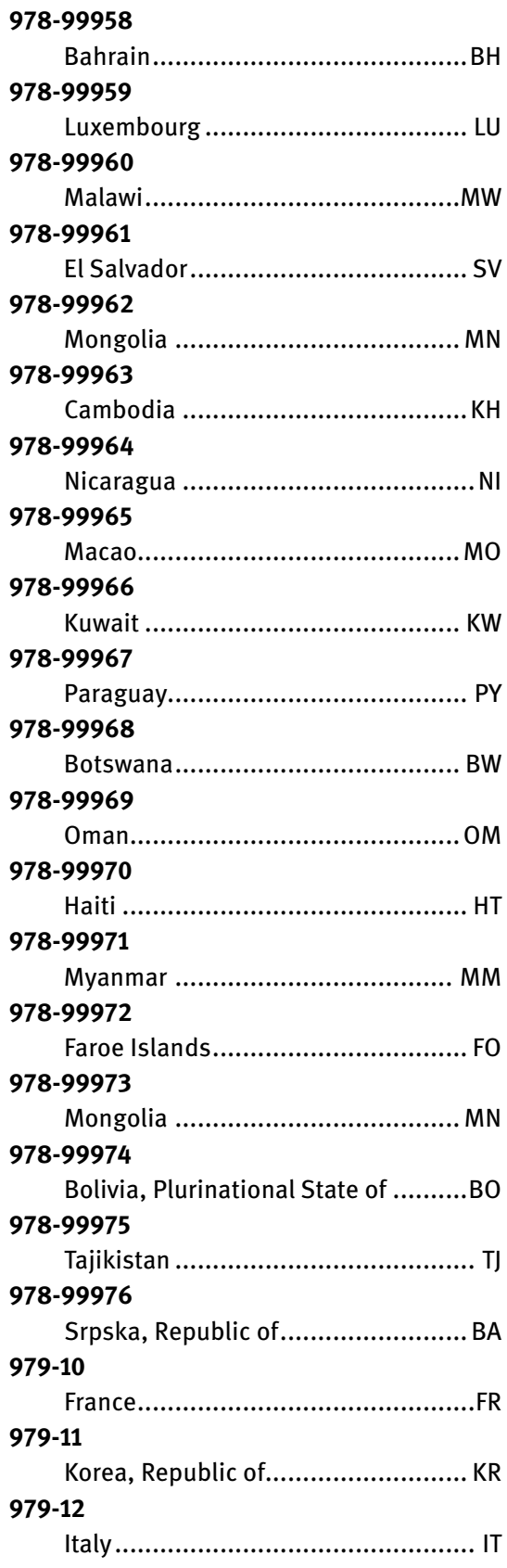

